\title{
AST/ALT ratio as a predictor of mortality and exacerbations of PM/DM-ILD in 1 year-a retrospective cohort study with 522 cases
}

\author{
Renjiao Li, Wen-Jun Zhu, Faping Wang, Xiaoju Tang and Fengming Luo ${ }^{*}(\mathbb{D}$
}

\begin{abstract}
Objective: To assess the associations between aspartate transaminase/alanine transaminase ratio (DRR) and mortality in patients with polymyositis/dermatomyositis-associated interstitial lung disease (PM/DM-ILD).

Patients and methods: This was a retrospective cohort study, which included 522 patients with PM/DM-ILD whose DRR on admission were tested at West China Hospital of Sichuan University during the period from January 1, 2008, to December 31, 2018. Cox regression models were used to estimate hazard ratios for mortality in four predefined DRR strata ( $\leq 0.91,0.91-1.26,1.26-1.73$, and $>1.73$ ), after adjusting for age, sex, DRR stratum, diagnosis, overlap syndrome, hemoglobin, platelet count, white blood cell count, the percentage of neutrophils, neutrophil/ lymphocyte ratio, albumin, creatine kinase, uric acid/creatinine ratio, triglycerides, or low-density lipoprotein.

Results: Higher DRR (> 1.73) was an independent predictor of 1-year mortality in multivariate Cox regression analysis (hazard ratio 3.423,95\% Cl 1.481-7.911, $p=.004$ ). Patients with higher DRR more often required the use of mechanical ventilation and readmission for acute exacerbation of PM/DM-ILD at 1-year follow-up.

Conclusion: Higher DRR on admission for PM/DM-ILD patients are associated with increased mortality, risk of mechanical ventilation, and hospitalization in 1-year follow-up. This low-cost, easy-to-obtain, rapidly measured biomarker may be useful in the identification of high-risk PM/DM-ILD patients that could benefit from intensive management.
\end{abstract}

Keywords: PM/DM, ILD, Mortality, Mechanical ventilation

\section{Introduction}

Polymyositis (PM) and dermatomyositis (DM) are common branches of connective tissue disease characterized by muscle weakness and skeletal muscle inflammation, which gradually involves other organs, especially the lungs [1]. Interstitial lung disease (ILD) is a major complication of $\mathrm{PM} / \mathrm{DM}$ with a prevalence of $20-78 \%$ according to studies [2-4]. ILD is also a negative prognostic factor

\footnotetext{
* Correspondence: fengmingluo@outlook.com

Department of Respiratory and Critical Care Medicine, West China Hospital, Sichuan University, 37 Guoxue Street, Chengdu 610041, Sichuan, China
}

associated with increased morbidity and mortality in patients with PM/DM [5-7]. An Italian study reported that the risk of mortality in patients with $\mathrm{PM} / \mathrm{DM}$-associated ILD (PM/DM-ILD) is 2.3 times to that in patients without ILD [8].

De Ritis ratio (DRR), the ratio of serum level of aspartate transaminase (AST) and alanine transaminase (ALT), was first described by Fernando De Ritis in 1957 [9]. AST and ALT are parts of the most common indicators of liver functions, which increase significantly during hepatocellular damage or death [10]. Over the last decades, DRR was

(c) The Author(s). 2020 Open Access This article is licensed under a Creative Commons Attribution 4.0 International License, which permits use, sharing, adaptation, distribution and reproduction in any medium or format, as long as you give appropriate credit to the original author(s) and the source, provide a link to the Creative Commons licence, and indicate if changes were made. The images or other third party material in this article are included in the article's Creative Commons licence, unless indicated otherwise in a credit line to the material. If material is not included in the article's Creative Commons licence and your intended use is not permitted by statutory regulation or exceeds the permitted use, you will need to obtain permission directly from the copyright holder. To view a copy of this licence, visit http://creativecommons.org/licenses/by/4.0/ The Creative Commons Public Domain Dedication waiver (http://creativecommons.org/publicdomain/zero/1.0/) applies to the data made available in this article, unless otherwise stated in a credit line to the data. 
considered as a useful indicator of liver diseases, such as hepatitis and liver cancers [11-13]. In recent years, increased levels of DRR have been shown in vascular disorders, including peripheral arterial occlusive disease [14], acute myocardial infarction [15], and acute ischemic stroke [16], and have been associated with malignant tumors $[17,18]$. Despite the above studies, to date, no study has evaluated the role of DRR on admission for PM/DM-ILD on the outcome of exacerbation and the long-term survival.

A number of studies have examined potential risk factors associated with poor survival in patients with $\operatorname{ILD}[19,20]$; however, little is known regarding the usefulness of biomarkers in PM/DM-ILD. Hence, the aim of this study was to identify this easy-to-obtain biomarker for assessing the disease activity of PM/DM-ILD.

\section{Methods}

This was a retrospective cohort study collecting secondary data from West China Hospital of Sichuan University. This study was approved by the Institutional Review Board of West China Hospital, Sichuan University, and written informed consent from patients was waived, but all analyses were carried out after anonymization of patients' data.

\section{Patients}

All patients who were diagnosed with PM/DM-ILD and had undergone AST and ALT measurement at West China Hospital during the period from January 1, 2008, to December 31, 2018, were eligible for inclusion in the study. All cases were diagnosed and assessed at the department of rheumatology or respiratory medicine. The diagnosis of PM/DM met the Bohan \& Peter Diagnostic Criteria [21] and the diagnosis of clinically amyopathic dermatomyositis (CADM) met the criteria developed by Sontheimer and colleagues [22]. Patients with acute hepatic failure or if they had suffered acute myocardial infarction which needed heart bypass surgery or percutaneous coronary intervention in 3 months were excluded. In addition, we excluded patients with chronic renal failure, especially dialysis patients, that significantly influence serum homeostasis, and patients with strenuous exercise within a week.

\section{Study design}

Patient demographics and laboratory and clinical parameters were collected from the medical record. Patient demographics included sex, age, and comorbidities, with special emphasis on hypertension, diabetes mellitus, and chronic hepatitis B. clinical parameters included ventilation, readmission, and diagnosis. Laboratory parameters included hemoglobin (HB), platelet count, white blood cell count (WBC), the percentage of neutrophils, lymphocytes, percentage of eosinophils (EO\%), albumin, AST, ALT, uric acid, creatinine, triglycerides, low-density lipoprotein, and creatine kinase (CK). Blood samples were collected from each patient at the time of admission to the emergency department or patients' fasting blood was obtained in the ward in the next morning, and all samples were sent to the clinical laboratory at West China Hospital.

Patients were evaluated on admission and were followed up for 1 year by monthly outpatient visits in the first 6 months and quarterly in the last 6 months. Routine clinical and laboratory examinations were included in those visits. Complete blood count, liver enzymes, and muscle enzymes were done every visit, and highresolution computed tomography was done every 3 months. The recording of mechanical ventilation (MV), noninvasive or invasive, was limited to hospital records of West China Hospital, and the recording of readmission for acute exacerbation of PM/DM-ILD (AEPM/DM-ILD) was limited to records of our hospital and confederate hospitals. Acute exacerbation was defined as worsening of dyspnea within 30 days, new radiographic opacities, the presence of new rash, recurrent muscle weakness, or elevated serum levels of muscle enzymes [23-26]. Survival data were retrieved from the electronic records of any accessible hospital in the southwest of China.

\section{Study outcomes}

The primary end-points were all-cause mortality at 1 year. Secondary outcomes included the need for MV and re-hospitalization for AEPM/DM-ILD in 1 year.

\section{Statistical analysis}

Summary statistics for normally distributed quantitative variables were expressed as means and standard deviations. For non-normally distributed variables, we use median and interquartile range. Categorical data were summarized by ratios and percentages. Comparisons between quantitative variables were analyzed by the Student's $t$, Mann-Whitney $U$ test, and Kruskal-Wallis tests based on variable distribution, and Bonferroni's multiple comparison tests were used for multiple group comparisons. Categorical variables were analyzed by chi-squared test or Fisher's exact test. Multicollinearity analysis using Spearman's correlation test $(r>0.5)$ was performed to identify the collinearity between the variables before further analysis.

For the analysis of the objectives, survival analyses and Cox regression analyses were implemented. In detail, we grouped the patients based on quartile of DRR at admission and used the first quartile as the reference group for all subsequent analyses. The times to death according to the DRR strata was evaluated with Kaplan-Meier survival curves and log-rank tests. We conducted univariate analysis by a univariate Cox regression model to assess the relationship between variables and outcomes. Then, we did a multivariable Cox regression to adjust potential confounders, 
and all variables with $P<0.1$ in the univariate analysis were subsequently entered into the model as potential predictors. All statistical analyses were performed with the IBM SPSS Statistical version 23.0 (IBM, Armonk, NY, USA SPSS) and graphs were drawn by Graphpad Prism 5.0 (Graphpad Software, La Jolla, CA, USA). A two-sided $p$ value less than 0.05 was considered statistically significant.

\section{Results}

\section{Patient characteristics}

A total of 522 patients were enrolled in the study. This cohort included 167 males (mean age $49.54 \pm 12.06$ years) and 355 females (mean age $50.09 \pm 11.25$ years). There were 360 (69\%), 47 (9\%), and 115 (22\%) patients who had DM-ILD, CADM-ILD, and PM-ILD, respectively. The prevalence of diabetes mellitus, hypertension, and chronic hepatitis B in this cohort was $6.9 \%(n=36)$, $10.3 \%(n=54)$, and $4.6 \%(n=24)$, respectively. Women had significantly higher median serum AST/ALT ratio and serum globin level than did men $(1.30(1-1.82)$ vs $1.12(0.77-1.56), p<0.001$; $31.7(27.6-35.9)$ vs $29.8(25.4-$ 33.7), $p=0.002$ ). Total bilirubin and direct bilirubin were lower in women in this study. Other clinical characteristics are shown in Table 1. Based on the physician's clinical

Table 1 Characteristics of all patients

\begin{tabular}{|c|c|c|c|c|}
\hline Characteristic & All $(n=522)$ & Male $(n=167)$ & Female $(n=355)$ & $p$ value \\
\hline Age, years & & $49.54(12.06)^{*}$ & $50.09(11.25)^{*}$ & 0.619 \\
\hline Diagnosis & & & & 0.085 \\
\hline DM & $360(69)$ & $126(75.4)$ & $234(65.9)$ & \\
\hline CADM & $47(9)$ & $11(6.6)$ & $36(10.1)$ & \\
\hline PM & $115(22)$ & $30(18)$ & $85(23.9)$ & \\
\hline OLS & & $9(5.4)$ & $41(11.5)$ & 0.026 \\
\hline \multicolumn{5}{|l|}{ Comorbidities } \\
\hline Diabetes mellitus & $36(6.9)$ & $13(7.8)$ & $23(6.5)$ & 0.583 \\
\hline Hypertension & $54(10.3)$ & $19(11.4)$ & $35(9.9)$ & 0.595 \\
\hline Chronic hepatitis B & $24(4.6)$ & $9(5.4)$ & $15(4.2)$ & 0.554 \\
\hline MDA5+ & $70(13.4)$ & $25(15)$ & $45(12.7)$ & 0.473 \\
\hline $\mathrm{HB}$ & & $130(120-146)$ & $121(109-131)$ & $<0.001$ \\
\hline PLT & & $194(143-254)$ & $195(148-262)$ & 0.508 \\
\hline $\mathrm{EO} \%$ & & $0.6(0.1-2.6)$ & $0.7(0.2-2.4)$ & 0.653 \\
\hline NLR & & $4.52(3.09-7.82)$ & $4.25(2.99-6.84)$ & 0.323 \\
\hline TBIL & & $9(6.8-12.3)$ & $8.4(6.5-11)$ & 0.042 \\
\hline DBIL & & $3.3(2.5-4.6)$ & $3(2.4-3.9)$ & 0.005 \\
\hline $\mathrm{IBIL}$ & & $5.2(4.1-7.8)$ & $5.5(4-6.9)$ & 0.441 \\
\hline TP & & $65.2(59.8-70.5)$ & $65.9(61.4-71.1)$ & 0.147 \\
\hline ALB & & $34.9(31.3-38.4)$ & $34.3(30.7-37.9)$ & 0.272 \\
\hline GLB & & $29.8(25.4-33.7)$ & $31.7(27.6-35.9)$ & 0.002 \\
\hline AST/ALT & & $1.12(0.77-1.56)$ & $1.30(1-1.82)$ & $<0.001$ \\
\hline UA/CREA & & $5.3(3.93-6.51)$ & $5.35(4.29-7.05)$ & 0.062 \\
\hline TG & & $1.82(1.35-2.51)$ & $1.97(1.45-2.79)$ & 0.016 \\
\hline LDL & & $2.46(1.81-3.05)$ & $2.29(1.68-3)$ & 0.221 \\
\hline CK & & $110(52-703)$ & $136(42-1156)$ & 0.943 \\
\hline Readmission within 1 year & & $36(21.6)$ & $67(18.9)$ & 0.472 \\
\hline Need for MV within 1 year & & $15(9)$ & $28(7.9)$ & 0.671 \\
\hline Deaths within 1 year & & $21(12.6)$ & $43(12.1)$ & 0.881 \\
\hline
\end{tabular}

Data are presented as $\mathrm{n}, \mathrm{n}(\%)$ for categorical variables and median (interquartile range) for continuous variables

*: mean (standard deviation)

Bold indicates statistical significance

$D M$ dermatomyosis, CADM clinically asymptomatic dermatomyosis, PM polymyosis, OLS overlap syndrome, MDA5 anti-melanoma differentiation-associated gene 5, $H B$ hemoglobin, PLT platelet count, WBC white blood cell count, EO\% percentage of eosinophils, NLR neutrophil/lymphocyte ratio, TBIL total bilirubin, DBIL direct bilirubin, IBIL indirect bilirubin, TP total protein, $A L B$ albumin, GLB globin, UA uric acid, CREA creatinine, $T G$ triglycerides, $L D L$ low-density lipoprotein, $C K$ creatine kinase, $M V$ mechanical ventilation 
experience, CS, CS pulse therapy, CYC, CSA, tacrolimus, MTX, AZA, MMF, IVIG, and other treatments were included in the induction treatment regimen. The results of collinearity were summarized in Additional file 1: Figure S1. There were not any variables in this study that highly correlated as indicated by Spearman's correlation test $(r>0.5)$.

\section{AST/ALT ratio (DRR) and associations with clinically relevant outcomes and mortality rates}

We further divided patients by DRR to explore the relative mortality risk across the DRR strata (Table 2).
Cumulative survival rates within 1 year across the DRR strata were $0.927,0.898,0.881$, and $0.733(p<0.001)$. Moreover, patients with higher DRR required MV and readmission within 1 year more often $(p<0.05)$. Figure 1 shows the cumulative survival curve of patients with different DRR strata. Apparently, higher DRR was associated with a poorer prognosis than lower DRR strata (stratum 1 vs stratum $4, p<0.001$; stratum 2 vs stratum 4 , $p=0.001$; stratum 3 vs stratum $4, p=0.006$ ), while the prognoses of patients with lower DRR strata did not differ.

Table 2 Clinical characteristics of patients across quartiles of AST/ALT ratio

\begin{tabular}{|c|c|c|c|c|c|}
\hline Characteristic & Group $1(\leq 0.91)$ & Group $2(0.91-1.26)$ & Group 3 (1.26-1.73) & Group 4 (> 1.73) & $p$ value \\
\hline $\bar{N}$ & 133 & 128 & 131 & 130 & \\
\hline Follow-up time (days) & $365(53-365)$ & $365(80.5-365)$ & $365(67-365)$ & $224(35-365)$ & 0.01 \\
\hline Sex, male & $58(43.6)$ & $38(29.7)$ & 44 (33.6) & $27(20.8)$ & 0.001 \\
\hline \multicolumn{6}{|l|}{ Age, years } \\
\hline$\leq 40$ & $31(23.3)$ & $20(15.6)$ & $28(21.4)$ & $22(16.9)$ & \multirow[t]{3}{*}{0.205} \\
\hline $40-60$ & $85(63.9)$ & $85(66.4)$ & $74(56.5)$ & $78(60)$ & \\
\hline$>60$ & $17(12.8)$ & $23(18)$ & $29(22.1)$ & $30(23.1)$ & \\
\hline \multicolumn{6}{|l|}{ Diagnosis } \\
\hline DM & $82(61.7)$ & $87(68)$ & $86(65.6)$ & $105(80.8)$ & \multirow[t]{3}{*}{0.046} \\
\hline CADM & $16(12)$ & $12(9.4)$ & $12(9.2)$ & $7(5.4)$ & \\
\hline PM & $35(26.3)$ & $29(22.7)$ & $33(25.2)$ & $18(13.8)$ & \\
\hline OLS & $11(8.3)$ & $12(9.4)$ & $16(12.2)$ & $11(8.5)$ & 0.681 \\
\hline \multicolumn{6}{|l|}{ Comorbidities } \\
\hline Diabetes mellitus & $8(6)$ & $11(8.6)$ & $8(6.1)$ & $9(6.9)$ & 0.834 \\
\hline Hypertension & $13(9.8)$ & $20(15.6)$ & $11(8.4)$ & $10(7.7)$ & 0.144 \\
\hline Chronic hepatitis B & $6(4.5)$ & $3(2.3)$ & $7(5.3)$ & $8(6.2)$ & 0.5 \\
\hline MDA5+ & $18(13.5)$ & $17(13.3)$ & $17(13)$ & $18(13.8)$ & 0.997 \\
\hline $\mathrm{HB}$ & $132(122.5-144.5)$ & $123(112-132)$ & $120(111-131)$ & $116.5(104.5-126)$ & $<0.001$ \\
\hline PLT & $192(140.5-233.5)$ & $194.5(144-262)$ & $195(154-261)$ & 196 (134.25-264.25) & 0.714 \\
\hline WBC & $8.07(6.2-10.63)$ & $6.92(4.68-10.52)$ & $5.89(4.49-8.05)$ & $5.41(3.97-7.81)$ & $<0.001$ \\
\hline $\mathrm{EO} \%$ & $0.3(0-0.9)$ & $0.75(0.3-2.58)$ & $1.6(0.3-3.5)$ & $1(0.18-2.83)$ & $<0.001$ \\
\hline NLR & $4.28(3.15-7.72)$ & $4.38(3.02-6.84)$ & $4.03(2.99-6.41)$ & $4.82(2.88-7.93)$ & 0.278 \\
\hline ALB & 36.2 (33.55-39.05) & 34.75 (31.93-38.48) & $34.3(30.9-38.3)$ & 32.15 (28.3-35.35) & $<0.001$ \\
\hline UA/CREA & $5(3.98-7.04)$ & $5.24(4.36-6.58)$ & $5.35(4.18-6.90)$ & $5.62(4.32-7.30)$ & 0.476 \\
\hline $\mathrm{TG}$ & $1.92(1.37-2.78)$ & $2.16(1.56-2.79)$ & $1.77(1.37-2.4)$ & $1.92(1.44-2.79)$ & 0.032 \\
\hline LDL & $2.86(2.3-3.52)$ & $2.44(1.89-3.11)$ & $2.24(1.64-2.77)$ & $1.86(1.32-2.53)$ & $<0.001$ \\
\hline CK & 69 (30.5-505.5) & $106.5(41-1786.25)$ & $177(67-1449)$ & 146.5 (59.75-992) & $<0.001$ \\
\hline \multicolumn{6}{|l|}{ Cumulative proportion } \\
\hline No readmission & 0.967 & 0.908 & 0.890 & 0.814 & 0.002 \\
\hline No need for MV & 0.944 & 0.942 & 0.92 & 0.805 & 0.003 \\
\hline Survival rate & 0.927 & 0.898 & 0.881 & 0.733 & $<0.001$ \\
\hline
\end{tabular}

Data are presented as $n, n$ (\%) for categorical variables and median (interquartile range) for continuous variables Bold indicates statistical significance

$D M$ dermatomyosis, CADM clinically asymptomatic dermatomyosis, PM polymyosis, OLS overlap syndrome, MDA5 anti-melanoma differentiation-associated gene 5 , $H B$ hemoglobin, PLT platelet count, WBC white blood cell count, EO\% percentage of eosinophils, NLR neutrophil/lymphocyte ratio, ALB albumin, UA uric acid, CREA creatinine, $T G$ triglycerides, $L D L$ low-density lipoprotein, $C K$ creatine kinase, $M V$ mechanical ventilation 


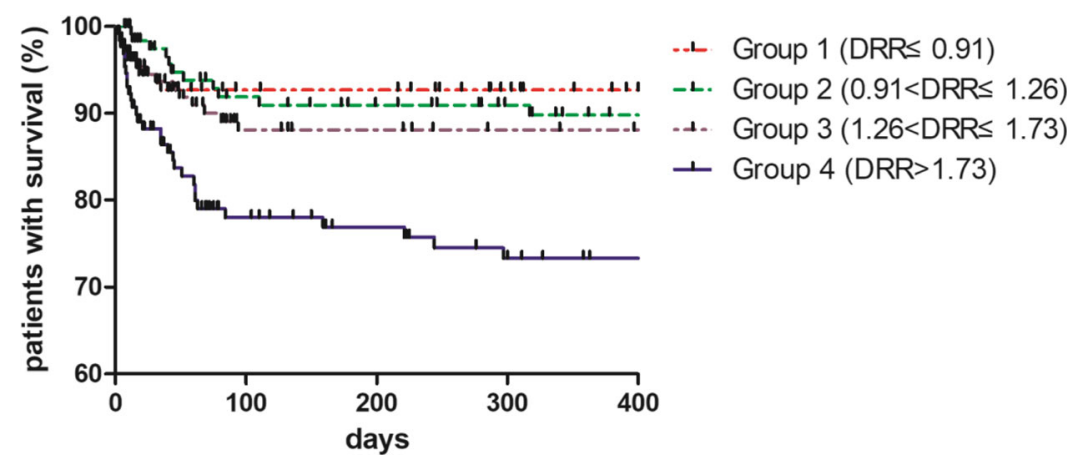

\begin{tabular}{lc}
\hline & Log Rank test p-value \\
\hline Group 1 VS Group 4 & $<0.001$ \\
Group 2 VS Group 4 & 0.001 \\
Group 3 VS Group 4 & 0.006 \\
\hline
\end{tabular}

Fig. 1 Kaplan-Meier survival analysis across quartiles of AST/ALT ratio

Table 3 shows the unadjusted hazard ratio (HR) for mortality and clinically relevant outcomes across the DRR strata, and Table 4 shows the HRs adjusted by different variables across the DRR strata. In general, the unadjusted HRs for readmission, MV, and all-cause mortality showed a J-shaped association across the DRR strata, which means high DRR stratum was associated with greater risks for readmission, MV, and mortality than low DRR strata. Using the DRR stratum 1 as a reference, the unadjusted HRs (95\% CIs) for readmission within 1 year were $1.00,1.454(0.794-2.662), 1.346$ (0.741-2.446), and 2.664 (1.513-4.692) across the DRR strata. Similarly, unadjusted HRs (95\% CIs) for MV within 1 year were $1.00,0.857 \quad(0.288-2.55), 1.429$ (0.544-3.756), and 3.195 (1.35-7.559). Unadjusted HRs (95\% CIs) for mortality within 1 year were $1.00,1.221$ $(0.506-2.94), 1.554(0.673-3.59)$, and 3.668 (1.7417.729).

As shown in Table 4, the adjusted HRs (95\% CI) for readmission, MV, and mortality also exhibited a Jshaped association across the DRR strata. The adjusted HRs (95\% CI) for readmission were 1.00, 1.830 (0.9813.412), 2.151 (1.151-4.020), and 4.546 (2.450-8.434); the adjusted HRs (95\% CI) for MV were 1.00, 1.086 (0.3563.312), 2.231 (0.791-6.294), and 4.172 (1.498-11.621); and the adjusted HRs $(95 \% \mathrm{CI})$ for 1-year mortality were $1.00,1.259$ (0.514-3.086), $1.814(0.757-4.35)$, and 3.423 (1.481-7.911).

\section{Discussion}

The present study investigated the association between DRR and mortality. Our analysis demonstrated a significant J-shaped association between DRR and all-cause mortality, with the lowest mortality occurring in individuals with DRR less than 0.91. Additionally, patients with increased DRR more often required the use of MV and readmission for AEPM/DM-ILD at 1-year follow-up. To our knowledge, this is the first study that has assessed DRR, a widely available and rapidly measured biomarker, as a predictor of clinically important outcomes in a retrospective cohort of patients with PM/DM-ILD.

Several mechanisms may be involved in the presence of high DRR in PM/DM-ILD. First, prolonged hypoxemia, caused by exacerbation of diffusing capacity for carbon monoxide (DLco), that is further increased with the development of ILD may result in increased pulmonary artery pressures, leading to increased right ventricle afterload, which promotes hepatocyte injury and AST level increased more [27]. Secondly, PM/DM is characterized by autoimmune conditions that target muscles to some degrees [28], and elevated serum CK, AST, and ALT levels have been associated with increased levels of injury to the skeletal muscle [27, 29]. The positive association of CK with the DRR in our cohort provides support for this mechanism. What is more, pulmonary infection, mainly bacterial, is the most common cause of acute exacerbation in patients with ILD. In the present study, almost all patients who complained of respiratory symptoms, such as cough and dyspnea, were confirmed suffering varying degrees of lung infection in highresolution computed tomography. Theoretically, serum infectious indicators in these patients, like WBC and neutrophil/lymphocyte ratio (NLR), would increase [30]. However, we found that the difference of WBC between DRR strata was statistically significant: WBC decreased with the increase of DRR, while that of NLR was not, which was a more sensitive marker in infectious diseases. A same trend with WBC was seen in $\mathrm{HB}$ as well. There is a possible explanation that patients in higher DRR strata present higher activity of autoimmunity, which attacks blood cells, with a decrease of WBC and HB. More studies are needed to confirm this hypothesis. 
Table 3 COX univariate analysis

\begin{tabular}{|c|c|c|c|c|c|c|}
\hline \multirow[t]{2}{*}{ Variables } & \multicolumn{2}{|c|}{ Readmission within 1 year } & \multicolumn{2}{|c|}{ Need for MV within 1 year } & \multicolumn{2}{|c|}{ Deaths within 1 year } \\
\hline & $p$ value & $\mathrm{HR}$ & $p$ value & $\mathrm{HR}$ & $p$ value & $\mathrm{HR}$ \\
\hline Sex & 0.249 & & 0.530 & & 0.700 & \\
\hline Age, years & 0.077 & & 0.042 & & 0.017 & \\
\hline \multicolumn{7}{|l|}{$\leq 40$} \\
\hline \multicolumn{7}{|l|}{$40-60(1)$} \\
\hline \multicolumn{7}{|l|}{$>60(2)$} \\
\hline Diagnosis & 0.028 & & 0.035 & & 0.093 & \\
\hline \multicolumn{7}{|l|}{$\mathrm{DM}$} \\
\hline \multicolumn{7}{|l|}{ ADM (1) } \\
\hline \multicolumn{7}{|l|}{ PM (2) } \\
\hline OLS & 0.102 & & 0.148 & & 0.078 & \\
\hline \multicolumn{7}{|l|}{ Comorbidities } \\
\hline Diabetes mellitus & 0.322 & & 0.395 & & 0.608 & \\
\hline Hypertension & 0.353 & & 0.785 & & 0.801 & \\
\hline Chronic hepatitis B & 0.233 & & 0.337 & & 0.998 & \\
\hline $\mathrm{HB}$ & 0.519 & & 0.062 & & 0.386 & \\
\hline PLT & 0.419 & & 0.598 & & 0.377 & \\
\hline WBC & 0.05 & & 0.631 & & 0.962 & \\
\hline $\mathrm{EO} \%$ & 0.017 & & 0.001 & & 0.017 & \\
\hline NLR & 0.514 & & $<0.001$ & & $<0.001$ & \\
\hline ALB & 0.842 & & $<0.001$ & & $<0.001$ & \\
\hline UA/CREA & 0.669 & & 0.003 & & 0.013 & \\
\hline AST/ALT & 0.003 & & 0.005 & & $<0.001$ & \\
\hline$\leq 0.91$ & Reference & & Reference & & Reference & \\
\hline $0.91-1.26(1)$ & 0.225 & 1.454 & 0.781 & 0.857 & 0.657 & 1.221 \\
\hline $1.26-1.73(2)$ & 0.329 & 1.346 & 0.469 & 1.429 & 0.302 & 1.554 \\
\hline$>1.73(3)$ & 0.001 & 2.664 & 0.008 & 3.195 & 0.001 & 3.668 \\
\hline $\mathrm{TG}$ & 0.011 & & 0.049 & & $<0.001$ & \\
\hline LDL & 0.152 & & 0.029 & & 0.149 & \\
\hline CK & 0.096 & & 0.082 & & 0.086 & \\
\hline
\end{tabular}

Bold indicates $p<0.1$

$D M$ dermatomyosis, CADM clinically asymptomatic dermatomyosis, $P M$ polymyosis, $O L S$ overlap syndrome, MDA5 anti-melanoma differentiation-associated gene 5 , $H B$ hemoglobin, PLT platelet count, WBC white blood cell count, EO\% percentage of eosinophils, NLR neutrophil/lymphocyte ratio, ALB albumin, UA uric acid, CREA creatinine, $T G$ triglycerides, $L D L$ low-density lipoprotein, $C K$ creatine kinase, $M V$ mechanical ventilation

Finally, ILDs comprise a large group of diseases that generally affect the interstitium [31] and have been associated with increased levels of eosinophils in bronchoalveolar lavage fluid and serum [32, 33], which work as cytokine mediators for acute and chronic inflammation, causing damage to the lung tissues, myocardium, skin, and gastrointestinal tract [34-37]. The positive association of EO\% with the DRR in our cohort provides support for this mechanism.

Numerous studies have attempted to identify biomarkers that predict clinically relevant outcomes for PM/DM-ILD. Higher levels of serum Chitinase-3-like-1 protein were negatively associated with DLco and prognoses of PM/DM-
ILD in a previous study [38]. In a Japanese study, Yasunori Enomoto et al. showed that higher soluble CD163 levels were associated with worse prognosis and forced volume vital percentage of predicted value [39]. Additionally, the measurement of several serum biomarkers, including Krebs von den Lungen-6 antigen [40], CD4+CXCR4+ T cells [41], microRNA-200c [42], soluble CD206 [43], and progranulin [44], succeeded to predict short-term or long-term prognosis in patients with PM/DM-ILD. The fact that high DDR acted as an independent predictor of 1-year mortality in our study suggests that this easy-toobtain biomarker may be used to identify high-risk patients that require more intensive treatment. 
Table 4 Cox multivariate regression analysis across quartiles of AST/ALT ratio

\begin{tabular}{|c|c|c|c|c|c|}
\hline AST/ALT ratio & $N(\%)$ & Unadjusted HR (95\%Cl) & $p$ value & Adjusted HR (95\%Cl) & $p$ value \\
\hline \multicolumn{6}{|l|}{ Mortality* } \\
\hline$\leq 0.91$ & $9(14.1)$ & Reference & & & \\
\hline $0.91-1.26$ & $11(17.2)$ & $1.221(0.506-2.947)$ & 0.657 & $1.259(0.514-3.086)$ & 0.614 \\
\hline $1.26-1.73$ & $14(21.9)$ & $1.554(0.673-3.590)$ & 0.302 & $1.814(0.757-4.35)$ & 0.182 \\
\hline$>1.73$ & $30(46.9)$ & $3.668(1.741-7.729)$ & 0.001 & $3.423(1.481-7.911)$ & 0.004 \\
\hline \multicolumn{6}{|c|}{ Mechanical ventilation** } \\
\hline$\leq 0.91$ & $7(16.3)$ & Reference & & & \\
\hline $0.91-1.26$ & $6(14)$ & $0.857(0.288-2.550)$ & 0.781 & $1.086(0.356-3.312)$ & 0.884 \\
\hline $1.26-1.73$ & $10(23.3)$ & $1.429(0.544-3.756)$ & 0.469 & $2.231(0.791-6.294)$ & 0.129 \\
\hline$>1.73$ & $20(46.5)$ & 3.195 (1.350-7.559) & 0.008 & $4.172(1.498-11.621)$ & 0.006 \\
\hline \multicolumn{6}{|l|}{ Readmission ${ }^{* * *}$} \\
\hline$\leq 0.91$ & $19(18.4)$ & Reference & & & \\
\hline $0.91-1.26$ & $25(24.3)$ & $1.454(0.794-2.662)$ & 0.225 & $1.62(0.879-3.066)$ & 0.120 \\
\hline $1.26-1.73$ & $26(25.2)$ & $1.346(0.741-2.446)$ & 0.329 & $1.935(1.020-3.673)$ & 0.043 \\
\hline$>1.73$ & $33(32)$ & $2.664(1.513-4.692)$ & 0.001 & 3.670 (1.968-6.844) & $<0.001$ \\
\hline
\end{tabular}

Bold indicates statistical significance

$N$ number, $H R$ hazard ratio, $C l$ confidence interval, $A S T$ aspartate transaminase, $A L T$ alanine transaminase

*HRs were adjusted by sex, age, diagnosis, OLS, EO\%, NLR, ALB, UA/CREA, TG, and CK

**HRs were adjusted by sex, age, diagnosis, HB, EO\%, NLR, ALB, UA/CREA, TG, LDL, and CK

***HRs were adjusted by sex, age, diagnosis, WBC, EO\%, TG, and CK

Previous studies have demonstrated higher DDR was associated with mortality in some malignant diseases, such as renal cell carcinoma, primary hepatic carcinoma, and upper tract urothelial cancer. A retrospective study reported significant associations between serum DRR and renal vein invasion, renal capsule infiltration, and renal pelvis involvement [45]. Another retrospective cohort study in 698 patients receiving nephrectomy has suggested that DRR is related to the prognosis of such patients, but the retrospective design may reduce the generalizability of the application of DRR [17]. And a study of 414 patients with primary hepatic carcinoma in China observed significant associations between DRR and mortality rate [46]. Although all the previously mentioned studies provide evidence that DRR is increased in more severe disease, no study had reported associations between DRR and PM/DM-ILD. In the present study, high DRR was an independent predictor of 1-year mortality in the multivariate Cox regression analysis, which suggests a possible role for this biomarker as a predictor of long-term mortality which needs to be evaluated in larger studies.

Autoimmune features in patients with ILD are important features in the natural history of the disease, and studies have reported that patients with inflammatory myopathies associated ILD have worse morbidity and higher mortality than patients without [47]. A retrospective cohort suggested that poorer pulmonary function test is more common in more severe PM/DM [48] and data from a nationwide prospective Pulmonary Hypertension Registry in France indicated a very possible association between inflammatory myopathies and pulmonary arterial hypertension [49]. This may, in part, explain the associations between high DRR with disease severity and mortality in the 1-year follow-up in our cohort. However, the fact that DRR remained an independent predictor of the risk for hospitalizations in the multivariate Cox regression analysis may suggest a possible role for this biomarker in the identification of exacerbationprone patients.

In the evaluation of AST and ALT levels, we need to take into account that these enzymes increase in a very sensitive but nonspecific way in several forms of tissue damage and inflammation, especially in muscle and liver tissues, all of which are very dynamic processes in patients with PM/DM-ILD [10]. AST and ALT are influenced by several factors including cardiovascular disease, food intake, exercise, renal dysfunction, and liver diseases. In the present study, we have excluded patients with chronic renal failure and acute hepatic failure, and we excluded patients with strenuous exercise within a week, but we cannot exclude other possible confounders that may have influenced our results. However, despite these possible limitations, we believe that the data from our cohort provide evidence for a possible role of DDR as a biomarker that is associated with disease severity and may identify patients with worse prognosis in hospitalized patients with PM/DM-ILD. A recent study showed that female is associated with poor outcomes in Chinese patients with PM/DM-ILD [50]. The predominance of female patients in stratum 4 of the present study 
also suggests that the elevated proportion of females may be associated with increased disease-related outcomes in our cohort. However, the fact that higher DDR continued to be an independent predictor of 1-year mortality, even after adjustment for the presence of females, further supports the possible role of DDR as a clinically relevant biomarker in PM/DM-ILD.

The present study presents some limitations. First, as in all retrospective cohort studies, the retrospective nature of data collection could not be avoided. And approximately a third of patients from the analysis were lost to follow-up within 1 year, of which 54 patients lost immediately after their discharge from the hospital. We decided to include them since this was an exploratory noninterventional study evaluating the possible role of DRR as a predictor of clinically relevant outcomes in patients with PM/DM-ILD which we wanted to maintain the integrity of these data and come to the conclusion as accurate as possible. Secondly, for the same reason, we were not able to collect high-quality data on cause-specific mortality. Finally, we do not have sufficient data about the readmission history of AEPM/DM-ILD in our population because of the TwoWay Referral Modes between Hospitals and Community Health Services in China, which restricts access for patients to our hospital, a first-class affiliated hospital. Hence, further studies with prospectively collected data are more appropriate in order to characterize patients as frequent exacerbators in the year of follow-up.

\section{Conclusion}

In conclusion, in the present study, we have shown that the AST/ALT ratio on admission for PM/DM-ILD is associated with increased 1-year mortality, increased risk of mechanical ventilation, and hospitalization for acute exacerbations of PM/DM-ILD in 1 year. Our results, combined with the fact that serum AST and ALT are widely and rapidly available, easy to interpret, low-cost biomarkers, suggest a possible role for AST/ALT ratio in the identification of PM/ DM-ILD patients at increased risk of adverse outcomes that may need early intensive management.

\section{Supplementary information}

Supplementary information accompanies this paper at https://doi.org/10. 1186/s13075-020-02286-w.

Additional file 1. Multicollinearity analysis using Spearman's correlation between main variables. HB: hemoglobin, PLT: platelet count, WBC: white blood cell count, NLR: neutrophil/lymphocyte ratio, EO: percentage of eosinophils, AST/ALT: aspartate transaminase/alanine transaminase ratio, ALB: albumin, UA/ CREA: uric acid/creatinine ratio, TG: triglycerides, LDL: low density lipoprotein, CK: creatine kinase.

\section{Abbreviations}

PM: Polymyositis; DM: Dermatomyositis; CADM: Clinically amyopathic dermatomyositis; ILD: Interstitial lung disease; PM/DM-ILD: Polymyositis/ dermatomyositis-associated interstitial lung disease; PM-ILD: Polymyositis- associated interstitial lung disease; DM-ILD: Dermatomyositis associated interstitial lung disease; CADM-ILD: Interstitial lung disease associated with clinically amyopathic dermatomyositis; DRR: De Ritis ratio; AST: Aspartate transaminase; ALT: Alanine transaminase; HB: Hemoglobin; WBC: White blood cell; EO\%: Percentage of eosinophils; CK: Creatine kinase; MV: Mechanical ventilation; AEPM/DM-ILD: Acute exacerbation of PM/DM-ILD; DLCO: Diffusing capacity for carbon monoxide; NLR: Neutrophil/lymphocyte ratio

\section{Acknowledgements}

Not applicable

\section{Authors' contributions}

Fengming Luo and Renjiao Li designed the study, analyzed all data, and wrote the manuscript. Faping Wang and Xiaoju Tang collected the experimental data and contributed to data evaluation. Wen-jun Zhu performed statistical analyses. All authors approved the version of the manuscript to be submitted. All authors take responsibility for the integrity of the data and the accuracy of the data analysis.

\section{Funding}

This work was supported by National Nature Science Foundation of China grant (NSFC No.81770072), "1.3.5 project for disciplines of excellence, West China Hospital, Sichuan University (ZYJC18021)", Chengdu Science and Technology Bureau (No. 2018-CY02-00064-GX), and National Key Research and Development Program (2017YFC0107805). The funders had no role in the study design, data collection and analysis, decision to publish, or preparation of the manuscript.

\section{Availability of data and materials}

The datasets used and analyzed during the current study are available from the corresponding author on reasonable request.

\section{Ethics approval and consent to participate}

This study was approved by Institutional Review Board of West China Hospital, Sichuan University.

\section{Consent for publication}

Not applicable.

\section{Competing interests}

The authors declare that they have no competing interests.

Received: 23 April 2020 Accepted: 5 August 2020

Published online: 20 September 2020

\section{References}

1. Dalakas MC, Hohlfeld R. Polymyositis and dermatomyositis. Lancet. 2003; 362(9388):971-82.

2. Mathai SC, Danoff SK. Management of interstitial lung disease associated with connective tissue disease. BMJ. 2016;352:h6819.

3. Chen IJ, Jan Wu YJ, Lin CW, Fan KW, Luo SF, Ho HH, et al. Interstitial lung disease in polymyositis and dermatomyositis. Clin Rheumatol. 2009;28(6): 639-46.

4. Hayashi S, Tanaka M, Kobayashi H, Nakazono T, Satoh T, Fukuno Y, et al. High-resolution computed tomography characterization of interstitial lung diseases in polymyositis/dermatomyositis. J Rheumatol. 2008;35(2):260-9.

5. Benbassat J, Gefel D, Larholt K, Sukenik S, Morgenstern V, Zlotnick A. Prognostic factors in polymyositis/dermatomyositis. A computer-assisted analysis of ninety-two cases. Arthritis Rheum. 1985;28(3):249-55.

6. Fathi M, Vikgren J, Boijsen M, Tylen U, Jorfeldt L, Tornling G, et al. Interstitial lung disease in polymyositis and dermatomyositis: longitudinal evaluation by pulmonary function and radiology. Arthritis Rheum. 2008;59(5):677-85.

7. Sugiyama Y, Yoshimi R, Tamura M, Takeno M, Kunishita Y, Kishimoto D, et al. The predictive prognostic factors for polymyositis/dermatomyositisassociated interstitial lung disease. Arthritis Res Ther. 2018;20(1):7.

8. Danieli MG, Gambini S, Pettinari L, Logullo F, Veronesi G, Gabrielli A. Impact of treatment on survival in polymyositis and dermatomyositis. A singlecentre long-term follow-up study. Autoimmun Rev. 2014;13(10):1048-54.

9. De Ritis F, Coltorti M, Giusti G. An enzymic test for the diagnosis of viral hepatitis; the transaminase serum activities. Clin Chim Acta. 1957;2(1):70-4. 
10. Botros M, Sikaris KA. The de ritis ratio: the test of time. Clin Biochem Rev. 2013;34(3):117-30.

11. Majhi S, Baral N, Lamsal M, Mehta KD. De Ritis ratio as diagnostic marker of alcoholic liver disease. Nepal Med Coll J. 2006;8(1):40-2.

12. Torkadi PP, Apte IC, Bhute AK. Biochemical evaluation of patients of alcoholic liver disease and non-alcoholic liver disease. Indian J Clin Biochem. 2014;29(1):79-83.

13. Darstein F, Hauser F, Straub BK, Wenzel JJ, Conradi R, Mittler J, et al. Hepatitis $E$ virus genotype 3 is a common finding in liver-transplanted patients undergoing liver biopsy for elevated liver enzymes with a low De Ritis ratio and suspected acute rejection: a real-world cohort. Clin Transpl. 2018;32(11):e13411.

14. Rief $P$, Pichler M, Raggam R, Hafner F, Gerger A, Eller $P$, et al. The AST/ALT (De-Ritis) ratio: a novel marker for critical limb ischemia in peripheral arterial occlusive disease patients. Medicine (Baltimore). 2016:95(24):e3843.

15. Steininger $M$, Winter MP, Reiberger $T$, Koller L, El-Hamid F, Forster $S$, et al. De-Ritis ratio improves long-term risk prediction after acute myocardial infarction. J Clin Med. 2018:7(12). PMID 30477196.

16. Gao F, Chen C, Lu J, Zheng J, Ma XC, Yuan XY, et al. De Ritis ratio (AST/ALT) as an independent predictor of poor outcome in patients with acute ischemic stroke. Neuropsychiatr Dis Treat. 2017;13:1551-7.

17. Bezan A, Mrsic E, Krieger D, Stojakovic T, Pummer K, Zigeuner R, et al. The preoperative AST/ALT (De Ritis) ratio represents a poor prognostic factor in a cohort of patients with nonmetastatic renal cell carcinoma. J Urol. 2015; 194(1):30-5

18. Lee H, Lee SE, Byun SS, Kim HH, Kwak C, Hong SK. De Ritis ratio (aspartate transaminase/alanine transaminase ratio) as a significant prognostic factor after surgical treatment in patients with clear-cell localized renal cell carcinoma: a propensity score-matched study. BJU Int. 2017;119(2):261-7.

19. Ohnishi H, Yokoyama A, Kondo K, Hamada H, Abe M, Nishimura K, et al. Comparative study of $K L-6$, surfactant protein-A, surfactant protein-D, and monocyte chemoattractant protein-1 as serum markers for interstitial lung diseases. Am J Respir Crit Care Med. 2002;165(3):378-81.

20. McFadden RG, Oliphant LD. Serum lactate dehydrogenase in interstitial lung disease. Chest. 1991;100(4):1182.

21. Bohan A, Peter JB. Polymyositis and dermatomyositis (first of two parts). N Engl J Med. 1975;292(7):344-7.

22. Sontheimer RD. Would a new name hasten the acceptance of amyopathic dermatomyositis (dermatomyositis sine myositis) as a distinctive subset within the idiopathic inflammatory dermatomyopathies spectrum of clinical illness? J Am Acad Dermatol. 2002;46(4):626-36.

23. An YS, Suh $\mathrm{CH}$, Jung JY, Kim HA. Role of bone scan in the assessment of polymyositis/dermatomyositis. Clin Rheumatol. 2015;34(4):699-706.

24. Collard HR, Moore BB, Flaherty KR, Brown KK, Kaner RJ, King TE Jr, et al. Acute exacerbations of idiopathic pulmonary fibrosis. Am J Respir Crit Care Med. 2007;176(7):636-43.

25. Schiffenbauer A, Garg M, Castro C, Pokrovnichka A, Joe G, Shrader J, et al. A randomized, double-blind, placebo-controlled trial of infliximab in refractory polymyositis and dermatomyositis. Semin Arthritis Rheum. 2018;47(6):858-64

26. Zhong Z, Lin F, Yang J, Zhang F, Zeng $X$, You X. Pregnancy in polymyositis or dermatomyositis: retrospective results from a tertiary centre in China. Rheumatology (Oxford). 2017;56(8):1272-5.

27. Dufour DR, Lott JA, Nolte FS, Gretch DR, Koff RS, Seeff LB. Diagnosis and monitoring of hepatic injury. I. Performance characteristics of laboratory tests. Clin Chem. 2000;46(12):2027-49.

28. Tieu J, Lundberg IE, Limaye V. Idiopathic inflammatory myositis. Best Pract Res Clin Rheumatol. 2016;30(1):149-68.

29. Radke JB, Algren DA, Chenoweth JA, Owen KP, Ford JB, Albertson TE, et al, Transaminase and creatine kinase ratios for differentiating delayed acetaminophen overdose from rhabdomyolysis. West J Emerg Med. 2018; 19(4):731-6

30. Russell CD, Parajuli A, Gale HJ, Bulteel NS, Schuetz P, de Jager CPC, et al. The utility of peripheral blood leucocyte ratios as biomarkers in infectious diseases: a systematic review and meta-analysis. J Inf Secur. 2019;78(5):339-48.

31. Wollin L, Distler JHW, Redente EF, Riches DWH, Stowasser S, SchlenkerHerceg $R$ et al: Potential of nintedanib in treatment of progressive fibrosing interstitial lung diseases. Eur Respir J 2019, 54(3). PMID 31285305.

32. Schroder T, Fuchss J, Schneider I, Stoltenburg-Didinger G, Hanisch F. Eosinophils in hereditary and inflammatory myopathies. Acta Myol. 2013;32(3):148-53.
33. Fujimoto K, Kubo K, Yamaguchi S, Honda T, Matsuzawa Y. Eosinophil activation in patients with pulmonary fibrosis. Chest. 1995;108(1):48-54.

34. Kovalszki A, Weller PF. Eosinophilia. Prim Care. 2016;43(4):607-17.

35. Kuang FL. Approach to patients with eosinophilia. Med Clin North Am. 2020;104(1):1-14.

36. Sheikh H, Siddiqui M, Uddin SMM, Haq A, Yaqoob U. The clinicopathological profile of eosinophilic myocarditis. Cureus. 2018;10(12):e3677.

37. Abedin N, Seemann T, Kleinfeld S, Ruehrup J, Roseler S, Trautwein C et al: Fecal eosinophil cationic protein is a diagnostic and predictive biomarker in young adults with inflammatory bowel disease. J Clin Med 2019, 8(12). PMID 31756948

38. Hozumi H, Fujisawa T, Enomoto N, Nakashima R, Enomoto Y, Suzuki Y, et al. Clinical utility of YKL-40 in polymyositis/dermatomyositis-associated interstitial lung disease. J Rheumatol. 2017;44(9):1394-401.

39. Enomoto $Y$, Suzuki $Y$, Hozumi H, Mori K, Kono M, Karayama M, et al. Clinical significance of soluble CD163 in polymyositis-related or dermatomyositisrelated interstitial lung disease. Arthritis Res Ther. 2017;19(1):9.

40. Takanashi S, Nishina N, Nakazawa M, Kaneko Y, Takeuchi T. Usefulness of serum Krebs von den Lungen-6 for the management of myositis-associated interstitial lung disease. Rheumatology (Oxford). 2019;58(6):1034-9.

41. Wang K, Zhao J, Chen Z, Li T, Tan X, Zheng Y, et al. CD4+CXCR4+ T cells as a novel prognostic biomarker in patients with idiopathic inflammatory myopathy-associated interstitial lung disease. Rheumatology (Oxford). 2019; 58(3):511-21.

42. Jiang Z, Tao JH, Zuo T, Li XM, Wang GS, Fang X, et al. The correlation between miR-200c and the severity of interstitial lung disease associated with different connective tissue diseases. Scand J Rheumatol. 2017;46(2):122-9.

43. Horiike Y, Suzuki Y, Fujisawa T, Yasui H, Karayama M, Hozumi H, et al. Successful classification of macrophage-mannose receptor CD206 in severity of anti-MDA5 antibody positive dermatomyositis associated ILD. Rheumatology (Oxford). 2019;58(12):2143-52.

44. Tanaka A, Tsukamoto H, Mitoma H, Kiyohara C, Ueda N, Ayano M, et al. Serum progranulin levels are elevated in dermatomyositis patients with acute interstitial lung disease, predicting prognosis. Arthritis Res Ther. 2015;17:27.

45. Canat L, Ataly HA, Agalarov S, Alkan I, Altunrende F. The effect of AST/ALT (De Ritis) ratio on survival and its relation to tumor histopathological variables in patients with localized renal cell carcinoma. Int Braz J Urol. 2018; 44(2):288-95.

46. Zhang $L X, L V Y, X u A M$, Wang $H Z$. The prognostic significance of serum gamma-glutamyltransferase levels and AST/ALT in primary hepatic carcinoma. BMC Cancer. 2019;19(1):841.

47. Cottin V, Thivolet-Bejui F, Reynaud-Gaubert M, Cadranel J, Delaval P, Ternamian PJ, et al. Interstitial lung disease in amyopathic dermatomyositis, dermatomyositis and polymyositis. Eur Respir J. 2003:22(2):245-50.

48. Kang EH, Lee EB, Shin KC, Im CH, Chung DH, Han SK, et al. Interstitial lung disease in patients with polymyositis, dermatomyositis and amyopathic dermatomyositis. Rheumatology (Oxford). 2005;44(10):1282-6.

49. Sanges S, Yelnik CM, Sitbon O, Benveniste O, Mariampillai K, PhillipsHoulbracq $M$, et al. Pulmonary arterial hypertension in idiopathic inflammatory myopathies: data from the French pulmonary hypertension registry and review of the literature. Medicine (Baltimore). 2016;95(39):e4911.

50. Yang X, Hao Y, Zhang X, Geng Y, Ji L, Li G, et al. Mortality of Chinese patients with polymyositis and dermatomyositis. Clin Rheumatol. 2020. PMID 31902027

\section{Publisher's Note}

Springer Nature remains neutral with regard to jurisdictional claims in published maps and institutional affiliations. 